\title{
On authorship in science: power, misconduct, responsibility and accountability
}

\author{
SAYANTAN DATTA
}

\begin{abstract}
The discovery of a case of data manipulation resulting in retraction of a high-impact paper revived conversations around scientific misconduct in India. Such malpractice is neither new nor rare. When it is discovered, there is a tendency to push the blame onto a junior author. But what makes one eligible to be an author in a scientific manuscript? In a case of misconduct, which authors must take the blame, and how do we hold them accountable? In this essay, I use the case of the recent retraction mentioned above to highlight the contentious nature of authorship in science.
\end{abstract}

Keywords: data manipulation, retraction, scientific misconduct, authorship, accountability

The modern-day practice of science is intricately linked to capitalism and productivity, and the author of a scientific text works within (and benefits from) these very frameworks of capitalism and productivity. Authors of a scientific text, especially in modern science, have certain gains to derive from the act of authorship. These gains can be in the form of better funding, secure faculty positions, etc [1,2].

When one looks at authorship in this context, one encounters two questions we continue to struggle with: One is the question of misconduct, and the other the question of shared responsibility. Instances when an author intentionally peddles a lie as truth, or when an author forges data or manipulates experiments and/or analysis to arrive at a desired conclusion, are on the rise. This is especially true in

Authors: Sayantan Datta, (sayantan@thelifeofscience.com), Faculty Teaching Associate, Centre for Writing and Pedagogy, Krea University, Andhra Pradesh, India; and Member, Editor and Contributor, TheLifeofScience.com.

To cite: Datta S. On authorship in science: power, misconduct, responsibility and accountability. Indian J Med Ethics. Published online first on February 23, 2022. DOI: 10.20529/IJME.2022.015

Manuscript Editor:Trudo Lemmens, Sandhya Srinivasan.

Peer Reviewers: Joe Varghese and an anonymous reviewer

\section{Copyright and license}

( I Indian Journal of Medical Ethics 2022: Open Access and Distributed under the Creative Commons license CC BY-NC-ND 4.0, which permits only non-commercial and non-modified sharing in any medium, provided the original author(s) and source are credited. the case of India. According to image forensics expert Elisabeth Bik, India and China are two countries from which manipulated or forged data are very common [3].

Moreover, one rarely encounters scientific texts that are authored by a single person these days. In a piece of work where there is more than one author, how is the responsibility for the integrity of the knowledge that the text holds, shared between authors? In the event of misconduct, data manipulation and forgery, who are the different authors to be held responsible? And, finally, how does one hold the authors accountable? Guidelines for authorship do exist, but are these guidelines implemented? More importantly, do these guidelines help us untangle the difficult nature of authorship in science?

These are not easy questions to answer, and I do not claim to be answering them in this piece. Instead, I present a case study to highlight the contentious nature of authorship in science, and to raise questions that need the scientific community's urgent attention. The case in question is that of the retraction of a study published by researchers from the National Centre for Biological Sciences (NCBS), part of the Tata Institute of Fundamental Research (TIFR), in the highimpact journal Nature Chemical Biology, the cause of the retraction being data manipulation and forgery [4]. In my capacity as a science journalist, I have extensively covered the retraction of this paper, the responses of the institution and of the principal investigator $(\mathrm{PI})$ to the retraction, and the events that followed $[5,6,7,8]$. Drawing from my analysis of these events, I will present a perspective of authorship in science that is inseparable from responsibility and accountability, and is intricately linked to the power structures and power hierarchies that guide the practice of science today.

\section{The NCBS retraction}

On October 5, 2020, a research article was published from the lab of Arati Ramesh, a principal investigator at the NCBS, Bengaluru, in Nature Chemical Biology. The paper, titled "Discovery of Iron-Sensing Bacterial Riboswitches", claimed to have found ribonucleic acid (RNA) molecules that can bind to iron and undergo conformational changes, which eventually leads to an expression of iron-related genes [4]. Simply put, the authors reported discovering a novel ironsensing molecule which could change gene expression in a biological entity. The paper was widely lauded [9]. 
However, in the same month, anonymous commenters on the discussion website PubPeer pointed out that the images in the paper had clear signs of forgery and falsification. Ramesh responded quickly, providing raw images of the acquired data to counter claims of manipulation. In her own words, "We have uploaded the raw images as obtained from the instruments so that you can rest your concerns. As you can see, we have not manipulated any images." [10]

Very soon, anonymous commenters on PubPeer discovered that the raw data itself showed signs of substantial falsification. The fraud was not limited to western blot and polyacrylamide gel electrophoresis (PAGE) data; people identified markers of fraud even in isothermal titration calorimetry (ITC) plots. Briefly, western blot analysis is a technique that allows biologists to separate and visualise proteins on a membrane. PAGE is a prerequisite to western blots and allows for a separation of proteins based on their mass. ITC allows researchers to quantify the interaction between two substances, often a small substance (eg iron) and a large molecule (eg RNA).

Ramesh responded once more, this time saying that they were "more than happy to share all the reagents, constructs and materials and a detailed protocol for ANY of these experiments so that anyone can corroborate the results of the experiments shown in the manuscript." [11] After PubPeer commenter Leucanella Acutissima pointed out that even if other scientists would be able to independently replicate these images, it wouldn't absolve the current authors of responsibility for their data; Ramesh stopped responding [12]. The paper was finally retracted on June 29, 2021 [4], less than a year after it was published, following an investigation by NCBS that involved a committee headed by an external member [13].

NCBS and Ramesh both came out with public statements after the retraction. The NCBS statement said, "The [investigation committee] report concluded that image manipulation[s] had indeed occurred, and were carried out by a single individual. This individual is no longer affiliated with NCBS. In accordance with the committee's recommendations, Dr Ramesh initiated the process of retracting the paper on February 1, 2021." [13] Ramesh's statement echoed the same sentiment when she said, "It was now unambiguous that some of the data had been manipulated. The specific data that were flagged came from one author, who left my lab abruptly within a few days after the investigation (without turning in the correct constructs/strains related to this project and without sharing some of the ITC raw data)." [14] Her statement did mention that she, as the corresponding author, took responsibility: "As the corresponding author, I must bear responsibility and am deeply shocked, disturbed and very saddened that such scientific misconduct could happen under my watch." [14]

Unfortunately, the statements turned out to be at least partly false. First, the student (and the first author of the paper), Siladitya Bandyopadhyay, did not leave abruptly [15]. In fact, the director of NCBS, Satyajit Mayor, accepted his resignation and he was granted a 'No Objection Certificate' by NCBS. Second, Bandyopadhyay alleged that Ramesh had access to all the raw data and constructs he had used for experiments [15]. Interestingly, Bandyopadhyay, while agreeing to his role in the fraud, also mentioned that he was not the only person to commit the fraud, and mentioned that the co-first author of the paper, Susmitnarayan Chaudhury, had assisted him in forging data. Moreover, according to Bandyopadhyay, Ramesh was not keen on retracting the paper initially [7]. Chaudhury, who had earlier taken to Facebook to defend Bandyopadhyay, soon turned against him [15].

The NCBS director, Satyajit Mayor, submitted the investigation report to the TIFR Academic Ethics Committee (TAEC), which started independently investigating both the retraction process and the integrity of the NCBS investigation. Conclusions from the TAEC report, made public on September 15, 2021, found several lapses in the details mentioned in the press releases by NCBS and Ramesh [16].

First, the TAEC concluded that indeed Bandyopadhyay was not the only one who had participated in the forgery; it concluded that Chaudhury was also a part of the fraud. Moreover, the report mentioned that Bandyopadhyay had already admitted to his part in the fraud, but did not mention whether Chaudhury owned up to his role. Setting the record straight, the report also asserted that "the statement in the press release of NCBS-TIFR that the malpractice was carried out by only one individual and the statement made by the PI on her website implying that one author had left her lab abruptly were both incorrect." [16]

Second, while highlighting that members of the Ramesh lab were "unanimous" [16] in saying that Ramesh would not have been a part of any unethical practice, the TAEC report also mentioned that the fact that Ramesh overlooked obvious signs of data manipulation counts as "scientific carelessness and lack of diligence." [16]

Among various other recommendations, two are worth noting here. First, the TAEC recommended that Chaudhury's current institution be made aware of the TAEC's finding that he had a part to play in the forgery [16]. Second, the TAEC recommended that a supervisory committee be set up to oversee general functioning and publication practices of Ramesh's lab, and that Ramesh be counselled "to be more professional in her scientific practices and her conduct in the laboratory." [16]

I have not mentioned all the details of the case since that is beyond the scope of this piece. Readers may wish to go through the cited reports to acquaint themselves with all the facts surrounding the case.

\section{Authorship in science: power}

Authorship in science must be understood within the 
context of power structures and power dynamics in the everyday practice of science. The principal investigator, or the group leader, of a particular laboratory has power over their trainees. Funding bodies have power over the kind of science that scientists can pursue. High-impact journals have power over what kind of science becomes popular and embedded in public consciousness. No exercise or relationship in science is devoid of a power dynamic.

Authorship in science often reflects this power hierarchy. At least in most disciplines of biology, the $\mathrm{PI}$ is usually the corresponding author of the paper, which means that all communication with respect to the paper should be addressed to the Pl. Papers from the same lab are often referred to with the PI's name. For example, if one were to refer to a body of knowledge produced over separate papers from somebody called X's lab, they would use the term "papers from X lab". Through this exercise, the PI emerges as the entity who is not just responsible for leading the production of knowledge that a particular text discusses, but who also appears in discussions following the codification of the knowledge produced in textual form.

In the same vein, the first author of a paper, often a graduate student or a postdoctoral fellow, has authority over the particular text that they produce. That text is often referred to by the name of the first author. For example, the case study presented above is about the "Bandyopadhyay et al." paper. Other authors of the paper are grouped into the "et al.", a term that literally means "and others". Many authors of a scientific text, then, are literally otherised in our references to scientific texts and their contribution to the study. Authorship in science, then, is in no way an egalitarian process. It is contingent on the productivity of the researcher in question, and the position they occupy on the academic ladder of science practice.

As we continue to think about the retraction of the paper from Ramesh's lab, another retraction in 2020 got scientists all over the world talking. This retraction, from the lab of 2018 Nobel Laureate Frances Arnold, was of a paper published in the journal Science in May 2019 [17]. In January 2020, Arnold took to Twitter to declare that owing to issues with reproducibility, they were retracting the paper [18]. In a separate tweet, she held herself accountable and responsible by saying that "I was a bit busy when this was submitted, and did not do my job well." [19] Both the tweets were met with an overwhelmingly positive response appreciating Arnold for taking responsibility and her pursuit of honesty.

One must ask about what Arnold did right to attract wide applause for retracting a paper that was not reproducible. I suggest that Arnold being a Nobel Laureate contributed significantly to how the scientific community responded to the retraction.

In fact, Science's retraction note does not reflect the same sentiment as Arnold's tweets [17]. The retraction note states that "efforts to reproduce the work showed that the enzymes do not catalyze the reactions with the activities and selectivities claimed. Careful examination of the first author's lab notebook then revealed missing contemporaneous entries and raw data for key experiments." [17] Here, it is clear that blame is laid on the first author of the paper for not maintaining an accurate record of raw data. The retraction note then, unlike Arnold's tweets, seems to pin the blame on the first author, much like Ramesh's and NCBS' press releases did.

Perhaps it is not fair to compare the retraction of the paper from Arnold's lab and that of the paper from Ramesh's lab. Ramesh is an early-career scientist, while Arnold is much more established and has a Nobel Prize to her credit. It would be interesting to know how the scientific community would have responded to a retraction from Arnold's lab had she been an early-career researcher and not a Nobel Laureate. Weishi Meng suggests in a blogpost that "the retraction would have been regarded as a red flag, motivating a thorough scrutiny of...Arnold's work in search for a misconduct pattern." [20]

It is important to note that unlike Ramesh's case, nobody suggested that Arnold be counselled, or work under a supervisory committee. The scientific community's responses to alleged misconduct and subsequent retractions of papers, then, seem to vary considerably depending on how powerful the principal researcher of the retracted paper is.

\section{Authorship in science: responsibility and accountability}

This brings us to the question of responsibility and accountability. As I have highlighted in the case study before, the first author of the paper - the graduate student in question - was held solely responsible for malpractice in the now-retracted paper. This is in no way an isolated incident. When CNR Rao, distinguished scientist and the then-scientific advisor to the Prime Minister, and a senior colleague of his, SB Krupanidhi, senior professor at the Indian Institute of Science, were accused of plagiarism in 2012, Krupanidhi told a reporter from The Hindu that "Senior authors mainly focus on experimental results, analysis and interpretation of results" and that although the responsibility for research misconduct lies with everybody, "when sharing the work in preparing the paper, the student should do the first draft, as it is part of training" [21]. Actually, by Krupanidhi's logic, the responsibility of correcting a draft falls on the senior authors, and that a paper was sent for publication (and managed to get published) without flagging plagiarism is indicative of a system that is failing, rather than the fault of one student.

More recently, when many papers co-authored by Gobardhan Das, an immunologist at the Jawaharlal Nehru University, were flagged on PubPeer for signs of forgery, he responded (while denying the allegations) by asserting that "there is nothing wrong with the images. In any case, the images are collected by students" [22]. There seems to be a trend here: Pls seem to be willing to shift the responsibility of misconduct on their students. This is a classic act of the 
more powerful exercising control over the less powerful.

What does this mean for the question of authorship? Let us look at the responsibilities of the varying levels of the hierarchy of authors in science. While there are various guidelines (for example, the International Committee for Medical Journal Editors has a set of guidelines for authorship) [23]; I have referred to the authorship guidelines of the Nature group of journals, since the journal in which the now-retracted paper by Bandyopadhyay et al was published belongs to the same group. According to the guidelines, one is eligible to be an author of a paper when one fulfils the following criteria:

- Each author is expected to have made substantial contributions to the conception or design of the work; or the acquisition, analysis, or interpretation of data; or the creation of new software used in the work; or have drafted the work or substantively revised it;

- AND to have approved the submitted version (and any substantially modified version that involves the author's contribution to the study);

- AND to have agreed both to be personally accountable for the author's own contributions and to ensure that questions related to the accuracy or integrity of any part of the work, even ones in which the author was not personally involved, are appropriately investigated, resolved, and the resolution documented in the literature [24].

In addition, the corresponding author is supposed to be responsible for the following:

- ensuring that data, materials, and code comply with transparency and reproducibility standards of the field and journal;

- ensuring that original data/materials/code upon which the submission is based are preserved following best practices in the field so that they are retrievable for reanalysis;

- confirming that data/materials/code presentation accurately reflects the original;

- foreseeing and minimising obstacles to the sharing of data/materials/code described in the work;

- ensuring that all authors (or group leaders in multi-lab collaborations) have certified the author list and author contributions [24].

If the PI followed these guidelines, isn't it surprising that Bandyopadhyay was the only one blamed by the PI and NCBS in the context of the retraction? This is especially striking when one looks at the contrast between which author gets accolades for their work and which author gets punished when the integrity of the work is called into question. When the paper was published, Ramesh as the corresponding author was conferred with praise. However, when questions concerning the integrity of the data were raised, things became more interesting. In her PubPeer responses, Ramesh did start with the use of "we", indicating shared responsibility in the work $[10,11]$. In her statement, she did hold herself responsible, but only at the level of supervision [14]. The burden of misconduct was entirely cast on Bandyopadhyay. This construction of Bandyopadhyay as the sole deviant from the otherwise pure pursuit of knowledge was crucial to the event that would be thought to resolve this crisis Bandyopadhyay's departure from the institute.

In fact, what is rather odd is that Ramesh pins the blame on Bandyopadhyay for not sharing raw data and constructs required for replication/reanalysis of the study. Interestingly, according to the Nature Authorship Guidelines above, it is not Bandyopadhyay's responsibility, but Ramesh's - she as the corresponding author is responsible for "ensuring that original data/materials/code upon which the submission is based are preserved following best practices in the field." [24]

The final point that I would like to deliberate on is the question of accountability. How do we hold an author accountable in cases of misconduct such as the one above? And which author(s) do we hold accountable?

The answers to these are not easy. Accountability has often been reduced to the act of taking responsibility. But holding someone accountable involves the person facing consequences for their actions. An apology is a good start, but probably not sufficient. This is simply because the corresponding author's apology to the scientific community does not compensate the student for the mental trauma they had to go through during their tenure in the lab; it doesn't protect a student from future judgement, and it doesn't provide the student a new space in this exercise of academic knowledge production.

My goal in this article was to highlight the nature of authorship in science as something that is inherently ill defined. I realise that in this deliberation on power, misconduct and authorship, I have raised complex questions rather than offering resolutions. Coming up with answers is an exercise in collective deliberation, with which I hope the science community in India will start engaging, rather than taking short-term measures to wash their hands of a responsibility that is ultimately for the entire ecosystem to bear.

\section{References}

1. Edwards MA, Roy S. Academic research in the 21 st century: maintaining scientific integrity in a climate of perverse incentives and hyper competition. Environ Eng Sci. 2017; 34, 51-61.

2. Day M. Capitalism is ruining science. The Wire Science. 2020 Mar 13 [cited 2021 Oct 26]. Available from: https://science.thewire.in/ science/capitalism-is-ruining-science/

3. Shen $\mathrm{H}$. Meet this super-spotter of duplicated images in science papers. Nature. 2020 May 8 [cited 2021 Oct 26]. Available from: https://www.nature.com/articles/d41586-020-01363-z

4. Bandyopadhyay S, Chaudhury S, Mehta D, Ramesh A. RETRACTED ARTICLE: Discovery of iron-sensing bacterial riboswitches. Nat Chem Biol. 2021 Aug 1 [cited 2021 Oct 26];17: 924. Available from https:// doi.org/10.1038/s41589-020-00665-7

5. Datta S. Some (volatile) thoughts on the recent data-forgery fiasco. sayantanspins.com. 2021 Jul 11 [cited 2021 Oct 26]. Available from: https://www.sayantanspins.com/post/recent-data-forgery-fiasco

6. Datta S. What the recent scientific misconduct episode at NCBS 
means for Indian science. TheLifeofScience.com. 2021 Jul 19 [cited 2021 Oct 26]. Available from: https://thelifeofscience.com/2021/07/19/ ncbs misconduct/

7. Datta S. NCBS Retraction: Ex-student alleges others involved in research fraud. The Wire Science. 2021 Jul 31 [cited 2021 Oct 26]. Available from: https://science.thewire.in/the-sciences/ncbsretraction-arati-ramesh-siladitya-bandyopadhyay-inquiry-committeeresearch-misconduct/

8. Datta S. TIFR probe draws the curtains on NCBS retraction case, but not all the way. The Wire Science. 2021 September 21 [cited 2021 Oct 26]. Available from: https://science.thewire.in/the-sciences/tifr-taecprobe-ncbs-arati-ramesh-retraction-case-supervisory-committee/

9. Article Metrics for RETRACTED ARTICLE: Discovery of iron-sensing bacterial riboswitches. Nature Chemical Biology. Date unknown [cited: 2021 Oct 26]. Available from: https://www.nature.com/articles/ s41589-020-00665-7/metrics.

10. Ramesh A. PubPeer response. PubPeer. 2020 Oct [cited 2021 Oct26]. Available from: https://pubpeer.com/publications/ CDC2E61BCA1A5D6FA70658F9CBBAE2\#3

11. Ramesh A. PubPeer response. PubPeer. 2020 Nov [cited 2021 Oct 26]. Available from:https://pubpeer.com/publications/ CDC2E61BCA1A5D6FA70658F9CBBAE2\#12

12. Acutissima L. PubPeer response. PubPeer. 2020 Nov [cited 2021 Oct 26]. Available from: https://pubpeer.com/publications/ CDC2E61BCA1A5D6FA70658F9CBBAE2\#13

13. National Centre for Biological Sciences. News. Retraction of paper from Arati Ramesh lab at NCBS. 2021 Jul 6 [cited 2021 Oct 26]. Available from: https://news.ncbs.res.in/research/retraction-paperarati-ramesh-lab-ncbs

14. Ramesh A. Personal Statement: Retraction of NCB 2020 paper. 2021 [cited 2021 Oct 26]. Available from: https://aratirameshlab.weebly.com/ uploads/4/3/3/2/43328125/personalstatement_retractionncb.pdf

15. Schneider L. "On no condition can the prediction be wrong" - NCBS Scapegoat Student in Interview. 2021 Aug 02 [cited 2021 Oct 26]. Available from: https://forbetterscience.com/2021/08/02/on-nocondition-can-the-prediction-be-wrong-ncbs-scapegoat-student-ininterview/

16. Press Release. Regarding recent allegations of academic malpractices at NCBS-TIFR. Date unknown [cited 2021 Oct 26]. Available from: https://www.tifr.res.in/TSN/article/Findings_of_the_TAEC.pdf

17. Cho I et al. Retraction: Site-selective enzymatic $\mathrm{C}-\mathrm{H}$ amidation for synthesis of diverse lactams. Science. 2020 Jan 10 [cited 2021 Oct 26]. Available from: https://www.science.org/doi/full/10.1126/ science.aba6100

18. Arnold F. Tweet. Twitter. 2020 [cited 2021 Oct 26]. Available from: https://twitter.com/francesarnold/status/1212796190711959552

19. Arnold F. Tweet. Twitter. 2020 [cited 2021 Oct 26]. Available from: https://twitter.com/francesarnold/status/1212796266494607360? ref_src=twsrc\%5Etfw\%7Ctwcamp\%5Etweetembed\%7Ctwterm\%5E $1212796266494607360 \% 7 C t w g r \% 5 E \% 7 C t w c o n \% 5 E s 1 \_\& r e f \_u r l=h t t$ ps\%3A\%2F\%2Fwww.rasayanika.com\%2F2020\%2F01\%2F07\%2Fno bel-laureate-retracts-paper-why-frances-h-arnold-retractedpaper\%2F

20. Meng W. Nobel prize winner Frances Arnold comes forward, retracts a paper and the post publication reporting idiocracy praises her. Science Transparency. 2020 Feb 28[cited 2021 Oct 26]. Available from: https://scienceretractions.wordpress.com/2020/02/28/nobelprize-winner-frances-arnold-comes-forward-retracts-a-paper-andthe-post-publication-reporting-idiocracy-praises-her/

21. Prasad R. More instances of plagiarism come to light. The Hindu. 2012, Updated 2016 [cited 2021 Oct 26]. Available from: https:// www.thehindu.com/news/national/more-instances-of-plagiarismcome-to-light/article2975271.ece

22. Basu M. 11 papers by JNU scientist \& BJP candidate Das flagged for 'manipulation', he blames politics. The Print. 2021 Aug 4[cited 2021 Oct 26]. Available from: https://theprint.in/science/11-papers-byjnu-scientist-bjp-candidate-das-flagged-for-manipulation-heblames-politics/709140/

23. International Committee for Medical Journal Editors. Defining the role of authors and contributors. ICMJE; Date unknown [cited 2021 Oct 26]. Available from: http://www.icmje.org/recommendations/ browse/roles-and-responsibilities/defining-the-role-of-authorsand-contributors.html

24. Nature Portfolio. Authorship. Nature. Date unknown [cited 2021 Oct 26]. Available from: https://www.nature.com/nature-portfolio/ editorial-policies/authorship 